\title{
The Potential of Developing Pan-Coronaviral Antibodies to Spike Peptides in Convalescent COVID-19 Patients
}

\author{
Andrii Rabets $^{1} \cdot$ Galyna Bila $^{1} \cdot$ Roman Grytsko $^{1} \cdot$ Markian Samborskyy $^{2}$ (D) Yuriy Rebets $^{2} \cdot$ Sandor G. Vari $^{3}$. \\ Quentin Pagneux ${ }^{4} \cdot$ Alexandre Barras $^{4} \cdot$ Rabah Boukherroub $^{4} \cdot$ Sabine Szunerits ${ }^{4} \cdot$ Rostyslav Bilyy $^{1}$ (i)
}

Received: 16 September 2020 / Accepted: 12 February 2021 / Published online: 6 March 2021

(c) L. Hirszfeld Institute of Immunology and Experimental Therapy, Wroclaw, Poland 2021

\begin{abstract}
Coronaviruses share conservative spike protein $(S)$ on their enveloped membrane surface, where $\mathrm{S} 1$ subunit recognizes and binds the cellular receptor, and the $\mathrm{S} 2$ subunit mediates membrane fusion. This similarity raises the question: does coronaviral infection by one create protection to others? Convalescent SARS-CoV-2 (COVID-19) sera were tested for cross reactivity with peptides from Middle East respiratory syndrome coronavirus (MERS-CoV) which shares $74 \%$ homology. Our results showed significant cross-reactivity with a peptide of the heptad repeat 2 (HR2) domain of the MERS-CoV spike protein. Sera samples of 47 validated seropositive convalescent COVID-19 patients and 40 sera samples of control patients, collected in pre-COVID time were used to establish cross-bind reactivity with the MERS-CoV peptide. Significantly stronger binding $(p<0.0001)$ was observed for IgG antibodies in convalescent COVID-19 patients compared to the control group. In ELISA, MERS-CoV peptide helps to discriminate post-COVID-19 populations and non-infected ones by the presence of antibodies in blood samples. This suggests that polyclonal antibodies established during SARS-CoV-2 infection can recognize and probably decrease severity of MERS-CoV and other coronaviral infections. The high homology of the spike protein domain also suggests that the opposite effect can be true: coronaviral infections produce cross-reactive antibodies effective against SARS-CoV-2. The collected data prove that despite the core HR2 region is hidden in the native viral conformation, its exposure during cell entry makes it highly immunogenic. Since inhibitory peptides to this region were previously described, this opens new possibilities in fighting coronaviral infections and developing vaccines effective even after possible viral mutations.
\end{abstract}

Keywords SARS-CoV-2 $\cdot$ COVID-9 $\cdot$ Coronavirus $\cdot$ Antibodies $\cdot$ Immunoglobulins

\section{Introduction}

Coronaviruses such as the Middle East respiratory syndrome coronavirus (MERS-CoV), Severe acute respiratory syndrome coronavirus (SARS-CoV-1), and the recently emerged SARS-CoV-2 are sharing several similar protein regions which are involved in the recognition of the host

Rostyslav Bilyy

r.bilyy@gmail.com

1 Danylo Halytsky Lviv National Medical University, Lviv, Ukraine

2 Explogen LLC, Lviv, Ukraine

3 International Research and Innovation in Medicine Program, Cedars-Sinai Medical Center, Los Angeles, CA, USA

4 University of Lille, CNRS, Centrale Lille, University of Polytechnique Hauts-de-France, UMR 8520, IEMN, Lille, France cells. The SARS-CoV-2 genome (30 kb in size) encodes a large, non-structural polyprotein [open reading frame (ORF) $1 \mathrm{a} / \mathrm{b}$ ] that is further proteolytically cleaved to generate $15 / 16$ proteins, four structural proteins, and five accessory proteins (ORF3a, ORF6, ORF7, ORF8 and ORF9). The four structural proteins consist of the spike (S) surface glycoprotein, the membrane $(\mathrm{M})$ protein, the envelope $(\mathrm{E})$ protein, and the nucleocapsid $(\mathrm{N})$ protein, which is essential for SARS-CoV-2 assembly and infection. The MERS-CoV genome structure is encoding 10 proteins; two replicase polyproteins (ORFs $1 \mathrm{ab}$ and 1a), three structural proteins (E, N, and M), a surface (spike) glycoprotein (S), and five non-structural proteins (ORFs 3, 4a, 4b, and 5) (Liu et al. 2004; Mackay and Arden 2015).

The spike surface glycoprotein (S) plays a key role in mediating virus attachment and fusion and is indeed present in all human infecting coronaviruses. They can be cleaved by host proteases into an $\mathrm{N}$-terminal $\mathrm{S} 1$ subunit 
and a membrane-bound $\mathrm{C}$-terminal $\mathrm{S} 2$ region. To engage a host receptor, the receptor-binding domain (RBD) of the $\mathrm{S} 1$ subunit undergoes conformational movements, which transiently hide or expose the determinants of receptor binding (Wrapp et al. 2020; Xia et al. 2019). The heptad repeat 1 (HR1) region in $\mathrm{S} 2$ subunits forms a homotrimeric structure, exposing three highly conserved hydrophobic grooves on the surface resulting in binding of three HR2 regions and the formation of a six-helix bundle (6-HB) structure. 6 -HB is responsible for a close approximation of viral and host membranes and their subsequent merging. Binding of HR1 and HR2 domains results in the 6-HB needed for merging with the host cell membrane. Thus targeting the HR2 domain specifically binding HR 1 could inhibit the viral cell entry (Xia et al. 2014). Further optimisation of the peptide sequence has also the potential of generating pan-coronaviral inhibitors, like EK1 (Xia et al. 2019) able to inhibit SARSCoV-2 pseudovirus infection (Xia et al. 2020).

The structure of HR2 is poorly resolved during crystallographic assessment due to a high level of flexibility (Yuan et al. 2017). Unlike highly mutable RBD, the HR1 and HR2 domains are highly conservative between coronaviruses, so form a perfect target for viral neutralization and generation of immunity that latter can be used for viral testing. Since HR2 and HR1 domains are merged and surface-exposed after $\mathrm{S}$ protein cleavage it is likely that these domains are highly immunogenic.

This similarity can result in the development of crossreactive antibodies and protection against other coronaviruses, in case of being infected by another virus species. In this work, we would like to establish if SARS-CoV-2 results in the production of antibodies, that are also recognizing MERS-CoV antigens.

\section{Materials and Methods}

\section{Patient Cohorts}

All analyses of human materials were performed following the institutional guidelines and with the approval of the Ethics Committees of Danylo Halytsky Lviv National Medical University (DH LNMU No. 5/2017-02-23). The current study involved 47 samples from SARS-CoV-2 infected patients treated in the Lviv Regional Clinical Infection Hospital of Infectious Disease/Department of Infectious Diseases, Danylo Halytsky Lviv National Medical University (Lviv, Ukraine) during April-May 2020. Written informed consent was obtained from every person before blood collection. All patients were PCR-positive upon hospitalization. Sera collection took place at least 3 weeks after the appearance of clinical symptoms when a person was recovered. Recovery was assessed by (a) disappearance of clinical symptoms, (b) two consecutive negative PCR tests made within 2 days difference. All sera collected were tested positive for anti-SARS-CoV-2 antibodies using reference detection test from Xema (Xema Medica, Ukraine/Finland), in this test a mixture of spike and nucleocapside antigens served as and antigen. Cohort of patients consisted from 29 males and 18 females, the disease course was mild in 11 persons $(23.4 \%)$, moderate in $28(59.6 \%)$ and severe in 8 cases $(17.0 \%)$; the later were associated with age $>60$ years old and accompanying chronic illnesses. For most cases incubation period lasted 5-14 days. None of the patients had a history of MERS-CoV infection.

A group of normal healthy donors (NHD), who have donated blood between June and November 2019 (preCOVID-19) served as controls, they were both age- and sexmatched to fit the study group. None of the healthy donors had a history of MERS-CoV infection. Informed written consent for blood withdrawal was obtained from each patient and NHD.

\section{ELISA Tests}

Sera samples from convalescent COVID-19 patients and NHDs were frozen at $-20{ }^{\circ} \mathrm{C}$. For testing anti-MERS response immunosorbent NUNC maxisorp plates (Thermo Scientific, Waltham, USA) were coated with $\mathrm{NH}_{2}$-CCT TTTTTSLTQINTTLLDLEYEMKKLEEVVKKLEESYIDLKEL-COOH peptide ( $50 \mu \mathrm{L}$ of a $4 \mu \mathrm{g} / \mathrm{mL}$ solution) in a carbonate-bicarbonate buffer (100 mM, pH 9.6). For testing anti-SARS-CoV-2 response immunosorbent NUNC MaxiSorp plates (Thermo Scientific, Waltham, USA) were coated with recombinant 194 a.a. protein corresponding to the RBD domain of spice protein of SARS-CoV-2, (Exploregen LLC, UA). All serum samples were diluted 1:1000 in the carbonate-bicarbonate buffer and incubated at $37{ }^{\circ} \mathrm{C}$ for $1 \mathrm{~h}$, after that the plates were washed again. Goat anti-human IgG (H + L)-horseradish peroxidase (109-035-003, Jackson ImmunoResearch) was diluted in washing buffer (1:25,000), added to the plates, and incubated at room temperature for $1 \mathrm{~h}$. After the corresponding washings, the assay was developed with 3,3',5,5'-tetramethylbenzidine (TMB) containing an excess of $\mathrm{H}_{2} \mathrm{O}_{2}$ as a substrate $(50 \mu \mathrm{L} /$ well $)$. The reaction was stopped with $50 \mu \mathrm{l} /$ well of sulfuric acid (1 M). The absorbance was read at $450 \mathrm{~nm} / 600 \mathrm{~nm}$ using a Perkin Elmer BioAssay reader HST700 (Waltham, USA). antiSARS-CoV-2 ELISA was additionally tested with reference positive sera for COVID-19 diagnostics, anth their signal was in the renge of $0.45-0.60$ optical density units. The coefficient of variation $(\mathrm{CV})$ between replicates was controlled to be $<3 \%$. Other ELISA parameters were controlled according to the best practice of ELISA analysis (Crowther 2009; Kiessig et al. 1993) and our previous reports (Biermann et al. 2018; Bozhenko et al. 2020). 


\section{Bioinformatics}

The protein homology searches were done using blast (National Center for Biotechnology Information and Protein Data Bank [PDB]) databases. To include the regions with resolved structures in our searches we had used SEQATOMS (http://www.bioinformatics.nl/tools/seqatoms/) (Brandt et al. 2008). Protein structures were visualized using PyMOL (https://pymol.org/). Multiple sequence alignments were done using CLUTALW (Larkin et al. 2007).

\section{Data Analysis}

ELISA testing was performed in duplicate using two technical replicates for each analysis (CV always $<3 \%$ ). The data were normalized between plates using positive controls and corrected for background signal of secondary antibodies on each plate, then the mean values were calculated. The mean values were used to construct data on the figures. For comparisons between two groups, the Mann-Whitney $U$-test for numerical variables was used. A receiver operating characteristic (ROC) curve was generated. The area under the ROC was calculated to estimate the specificity, sensitivity, and usefulness of the binding assays. All analyses were performed using Excel 2016 (Microsoft Corp., Redmond, WA, USA) and Prism 8.2 (GraphPad, San Diego, USA) software. A $p$ value of $\leq 0.05$ was considered statistically significant. Four levels of significance are depicted in the figures by asterisks: ${ }^{*} p<0.05 ; * * p<0.01 ; * * * p<0.001$; $* * * * p<0.0001$.

\section{Results}

\section{Selection's Rationale of S1 MERS Peptide}

The complete crystal structure of the HR2 domain of SARSCoV-2 remains currently unavailable due to its conformation changes and problems in stabilization (Wrapp et al. 2020) (Fig. 1a). Using the SEQATOMS algorithm (Brandt et al. 2008) we selected the most complete structure of the corresponding region containing defined atomic coordinates, namely one proposed by Walls et al. (2017), a model for HR1 HR2 rearrangements and unfolding accompanying viral entry into host cells for other coronaviruses. This structure demonstrates exposure of the HR2 domains upon cellular binding in trimeric (Fig. 1b) and monomeric form (Fig. 1c). To evaluate the cross-reactivity we selected the HR2-specific peptide of the spike protein of MERS-CoV reported to possessed high immunogenic potential (Du et al. 2013; Mou et al. 2013; Wang et al. 2013). Indeed, ongoing studies show that it can be also successfully used to raise MERS-recognizing antibodies in the presence of neutrophil extracellular traps (NET) forming nanoadjuvants (Bilyy et al. 2019). The selected HR2 peptide of the spike protein of MERS-CoV [depicted yellow, Fig. 1d using PDB deposited crystal structure of 4NJL_A, (Lu et al. 2014)] shared significant similarities in 3D structure between (the only) known crystal structure of unfolded HR2 domain (Fig. 1c) and with pan-coronaviral inhibitor peptide EK1, using crystal structure 5ZVK_a (Xia et al. 2019) (Fig. 1e). Performed protein BLAST analysis revealed $46 \%$ identity and $76 \%$ similarity in amino acid sequence of the MERS peptide with the corresponding peptide of the SARS-CoV-2 spike protein (sequence ID QKJ68605.1) (Fig. 1f). The obtained data of closed structural similarity between viral HR2 domains of $\mathrm{S}$ protein inspired us to permorm ELISA tests for crossreactivity of produced antibodies.

\section{Antibody Cross-Reactivity towards Coronaviral HR2 Domains}

Having established the structural similarity between the S1 MERS peptide with the genome of SARS-CoV-2, sera of convalescent SARS-CoV-2 infected patient, who have never suffered from MERS-CoV infection before, have been collected and tested for the presence of antibodies. The S1 MERS-CoV specific peptide, $\mathrm{NH}_{2}$-CCTTTTTTSLTQINTTLLDLEYEMKKLEEVVKKLEESYIDLKEL-COOH, which we previously successfully used to raise anti-MERS antibodies while testing novel NET-stimulating adjuvants (Bilyy et al. 2019), was immobilized on ELISA plates and incubated with sera samples. As can be seen from Fig. 2a, stronger binding of IgG from sera of SARS-CoV-2 convalescent patients is observed when compared to sera of patients without previous SARS-CoV-2 infection $(p<0.0001$, $n=87)$. Minor differences were detected for IgM binding $(p=0.016)$ and no difference was detected for IgA or IgE antibody subclasses (Supplementary Information Fig. S1).

Using this S1 MERS-CoV specific peptide, discrimination of persons that have suffered from SARS-CoV-2 infection and those who were not in contact with the virus resulted in a predictive value (area under ROC curve) equal to 0.823 (Fig. 2b), with a specificity and sensitivity of $\sim 60 \%$ (95\% confidence). SARS-CoV-2 infection results in the generation of antibodies with significantly strong cross-reactive towards a MERS-specific peptide with 76\% homology. Highly conservative regions of the exposed domain suggest that the opposite can be true-the coronaviral disease can result in some antibodies able to recognize SARS-CoV-2 epitops circulating in the blood.

To determine whether the strong binding with $\mathrm{S} 1$ peptide is correlated with a higher amount of anti-SARS-CoV-2 antibodies we used recombinant RBD protein immobilization on ELISA plates to incubate with sera samples to evaluate the amount of formed IgG type antibodies. As can be seen from 

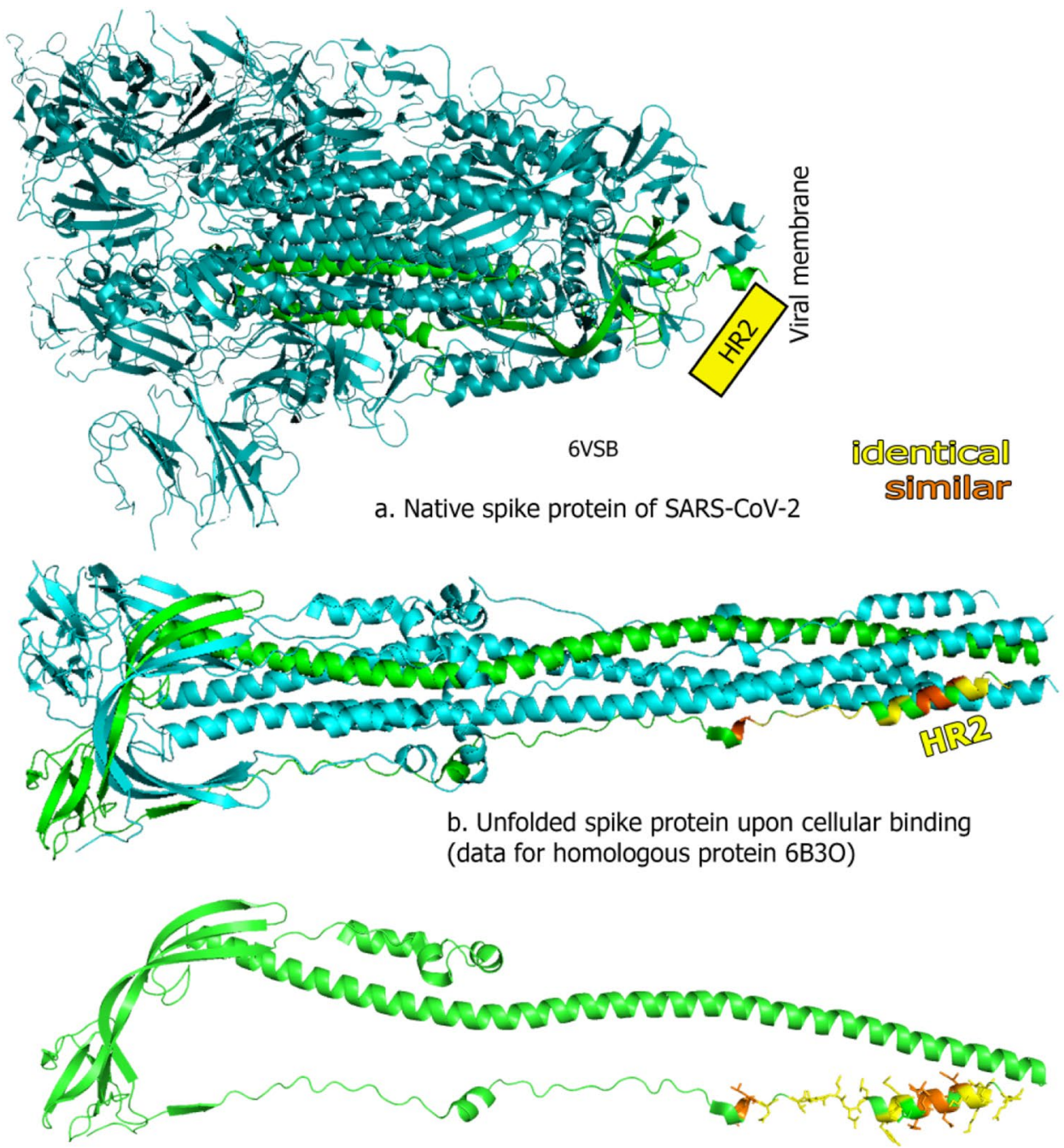

c. Immunogenic region in the unfolded spike protein demonstrated in a monomer of 6B30_A

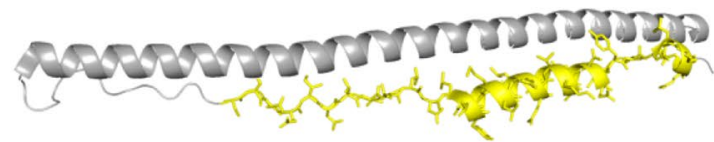

d. MERS-CoV peptide used for testing (yellow) visualized in 4NJL_A

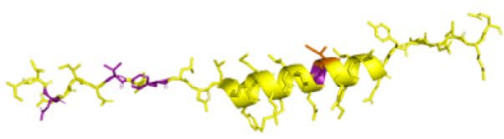

e. Pan-coronaviral peptide inhibitor EK1 5ZVK_a

f.

\begin{tabular}{llllll}
\hline Score & Expect & Method & Identities & Positives & Gaps \\
37.7 bits(86) & $2 \mathrm{e}-04$ & Composition-based stats. & $16 / 35(46 \%)$ & $26 / 35(74 \%)$ & $0 / 35(0 \%)$ \\
\hline
\end{tabular}

Query 2 LTQINTTLLDLEYEMKKLEEWVKKLEESYIDLKEL 36

$\begin{array}{llll}\text { Sbjet } 1169 & \stackrel{++}{++} \text { IN }+++++++E++ \text { L EV K L ES IDLKEL } & \\ \text { ISGINASWNIQKEIDRLNEVAKNLNESLIDLKEL } & 1203\end{array}$ 
४Fig. 1 Similarity of HR2 regions between different coronaviruses. a Image of Spike protein (S1) of SARS-CoV-2. b S1 upon host cell interaction: conformational changes in trimer are occurring, exposing previously hidden HR2 domain regions (Wrapp et al. 2020), c monomeric part of (b), exposing domain with structural similarity (yellow-identical, orange-similar amino acids) towards corresponding MERS peptide, depicted on (d) and pan. (f). Protein BLAST analysis of used MERS protein is showing similarity towards the sequence in the genome of SARS-CoV-19. Sequence ID: QKJ68605.1. Crystal structure of HR2 domain for SARS-CoV-2 is currently not available, thus it is represented as a rectangle in (a) based on the last connected coordinates available in 6VSB structure

Fig. 2c, the sera samples having shown stronger binding of IgG antibodies with anti-HR2 MERS spike protein also contained higher IgG reactivity towards anti-RBD spike protein of SARS-CoV-2. The Pearson's correlation between the two parameters was $0.5492, p<0.0001$. This suggests stronger humoral response towards one of the virus will be associated with the intensity of the immune response towards other coronaviruses.

\section{Discussion}

Cross-reactivity between coronaviruses has become a critical question since it brings new promises against COVID19 protection. On the other hand, this cross-reactivity can be negative, since available cross-reactivity towards coronaviruses will make coronaviruses not the best choice for vectors in vaccines, especially taking into account recent data on broad immune cross-reactivity ( $\mathrm{Ng}$ et al. 2020).
Indeed, it was reported that epitope pools detect $\mathrm{CD} 4^{+}$and $\mathrm{CD}^{+} \mathrm{T}$ cells in $100 \%$ and $70 \%$ of convalescent COVID-19 patients respectively, recognizing $\mathrm{S}$ and $\mathrm{M}$ proteins, with at least eight SARS-CoV-2 ORFs targeted. T cell reactivity to SARS-CoV-2 epitopes is also detected in non-exposed individuals (Grifoni et al. 2020; Mateus et al. 2020). In SARS-CoV-2 patients, S-reactive $\mathrm{CD} 4^{+} \mathrm{T}$ cells equally target $\mathrm{N}$-terminal and $\mathrm{C}$-terminal parts of the spike protein, whereas in healthy donors $\mathrm{S}$-reactive $\mathrm{CD} 4^{+} \mathrm{T}$ cells react almost exclusively to the $\mathrm{C}$-terminal part. This part is characterized by a higher homology to spike glycoprotein of human endemic "common cold" coronaviruses and contains the $\mathrm{S} 2$ subunit of $\mathrm{S}$ with the cytoplasmic peptide, the fusion peptide, and the transmembrane domain but not the RBD. S-reactive $\mathrm{CD} 4^{+} \mathrm{T}$ cells from SARS-CoV-2 patients are further distinct to those from healthy donors as they coexpressed higher levels of CD38 and HLA-DR, indicating their recent in vivo activation (Braun et al. 2020). Potential preexisting cross-reactive $\mathrm{T}$ cell immunity to SARS-CoV-2 has broad implications, as it could explain aspects of differential SARS-CoV-2 clinical outcomes, influence epidemiological models of herd immunity, or affect the performance of SARS-CoV-2 candidate vaccines. Pre-existing memory $\mathrm{CD} 4^{+} \mathrm{T}$ cells that are cross-reactive with comparable affinity to SARS-CoV-2 and the common cold coronaviruses $\mathrm{HCoV}$ OC43, HCoV-229E, HCoV-NL63, or HCoV-HKU1. Thus, variegated $\mathrm{T}$ cell memory to coronaviruses that cause the common cold may underline at least some of the extensive heterogeneity observed in COVID-19 disease (Mateus et al. 2020). a

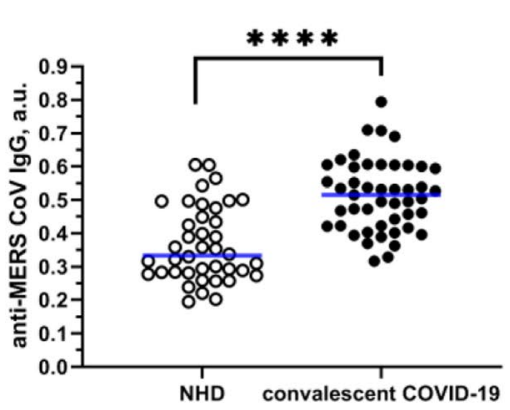

b

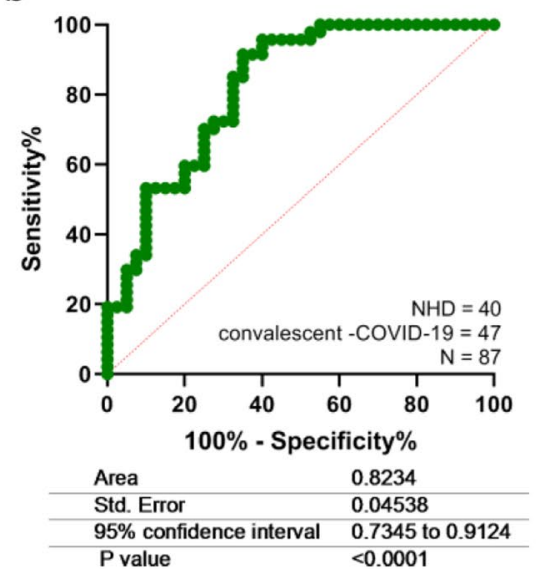

C

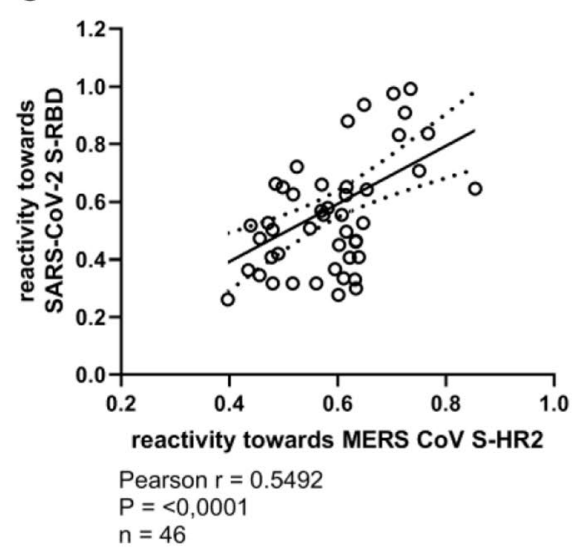

Fig. 2 Antibody cross-reactivity between coronaviruses: sera of convalescent COVID-19 patients possess antibodies recognizing MERSspecific peptide of HR2 spike protein domain. a Difference between $\mathrm{IgG}$ levels recognizing peptide of HR2 domain in S protein of MERS in convalescent SARS-CoV-2 patients and non-infected healthy donors. b ROC curve for discrimination of "anti-coronaviral" IgG antibodies using indicated MERS peptide as antigen. c IgG reactivity in convalescent plasma of SARS-CoV-2 patients towards HR2 domain in $\mathrm{S}$ protein of MERS vs RBD part of $\mathrm{S}$ protein of SARS$\mathrm{CoV}-2 . * * * * p<0.0001$ 
Based on our data and those of others, it would be reasonable to assume that the exposure of the C-terminal of the spike protein upon merging with the host cell makes it an excellent immunogenic target. It conservative nature could also allow the development of cross-viral immunity (cellular and humoral) which circumvent the high mutation rate in regions such as ACE2.

Our study was performed on the cohort of 47 patients, thus more detailed information for cross-coronaviral immunity against different strains is needed. However, after the current report was published as preprint (Rabets et al. 2020), there were a series of works confirming our idea, that humoral cross-coronaviral immunity is important in defining COVID (Beretta et al. 2020; Lipsitch et al. 2020; Yaqinud$\operatorname{din} 2020)$.

Supplementary Information The online version contains supplementary material available at https://doi.org/10.1007/s00005-021-00607-8.

Acknowledgements We thank Prof. Jean Dubuission and Dr. Karin Seron, University of Lille, CNRS, INSERM, CHU Lille, Institut Pasteur de Lille, U1019-UMR 8204-CIIL-Center for Infection and Immunity of Lille, F-59000 Lille, France for providing immunogenic MERS peptides amd Dr. Yuri Lebedin and Xema Medica Co, Ukraine/Finland for providing refence sera and tests. Financial support from the CedarsSinai Medical Center's International Research and Innovation in Medicine Program, the Association for Regional Cooperation in the Fields of Health, Science and Technology (RECOOP HST Association) RCSS grant 020, and BMYSRG 015; Grant of Ministry of Healthcare of Ukraine 0119U101338 and National Research Foundation of Ukraine 2020.02/0131; Volkswagen-Stiftung grant No 97744. This project has received funding from the European Union's Horizon 2020 research and innovation programme under Grant agreements No 861878 and 872331. Financial support by ANR project "nanoMERS" (ANR-

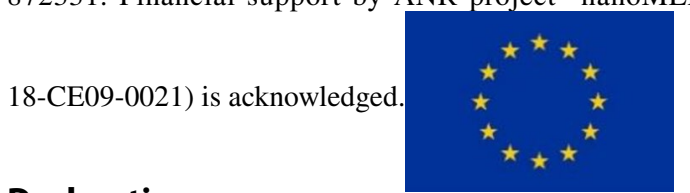

\section{Declarations}

Conflicts of Interest The authors declare no conflict of interest.

\section{References}

Beretta A, Cranage M, Zipeto D (2020) Is cross-reactive immunity triggering COVID-19 immunopathogenesis? Front Immunol 11:567710. https://doi.org/10.3389/fimmu.2020.567710

Biermann MHC, Boeltz S, Pieterse E et al (2018) Autoantibodies recognizing secondary necrotic cells promote neutrophilic phagocytosis and identify patients with systemic lupus erythematosus. Front Immunol 9:989. https://doi.org/10.3389/fimmu.2018.00989

Bilyy R, Paryzhak S, Turcheniuk K et al (2019) Aluminum oxide nanowires as safe and effective adjuvants for next-generation vaccines. Mater Today 22:58-66. https://doi.org/10.1016/j.matto d.2018.10.034

Bozhenko M, Boichuk M, Bila G et al (2020) Freezing influences, the exposure of $\mathrm{IgG}$ glycans in sera from multiple sclerosis patients. Ukr Biochem J 92:21-31. https://doi.org/10.15407/ubj92.02.021
Brandt BW, Heringa J, Leunissen JAM (2008) SEQATOMS: a web tool for identifying missing regions in PDB in sequence context. Nucleic Acids Res 36:W255-W259. https://doi.org/10.1093/nar/ gkn237

Braun J, Loyal L, Frentsch M et al (2020) (2020) Presence of SARS-CoV-2 reactive T cells in COVID-19 patients and healthy donors. MedRxiv 04(17):20061440. https://doi. org/10.1101/2020.04.17.20061440

Crowther JR (2009) The ELISA guidebook. Humana Press. https://doi. org/10.1007/978-1-60327-254-4

Du L, Zhao G, Kou Z et al (2013) Identification of a receptor-binding domain in the S Protein of the novel human coronavirus middle east respiratory syndrome coronavirus as an essential target for vaccine development. J Virol 87:9939-9942. https://doi. org/10.1128/JVI.01048-13

Grifoni A, Weiskopf D, Ramirez SI et al (2020) Targets of T cell responses to SARS-CoV-2 coronavirus in humans with COVID19 disease and unexposed individuals. Cell 181:1489-1501.e15. https://doi.org/10.1016/j.cell.2020.05.015

Kiessig S, Abel U, Risse P et al (1993) Problems of cut-off level determination in enzyme immunoassays: The case of TBE-ELISA. Klin Lab 39:877-886

Larkin MA, Blackshields G, Brown NP et al (2007) Clustal W and clustal X version 2.0. Bioinformatics 23:2947-2948. https://doi. org/10.1093/bioinformatics/btm404

Lipsitch M, Grad YH, Sette A et al (2020) Cross-reactive memory $\mathrm{T}$ cells and herd immunity to SARS-CoV-2. Nat Rev Immunol 20:709-713. https://doi.org/10.1038/s41577-020-00460-4

Liu S, Xiao G, Chen Y et al (2004) Interaction between heptad repeat 1 and 2 regions in spike protein of SARS-associated coronavirus: implications for virus fusogenic mechanism and identification of fusion inhibitors. Lancet 363:938-947. https://doi. org/10.1016/S0140-6736(04)15788-7

Lu L, Liu Q, Zhu Y et al (2014) Structure-based discovery of Middle East respiratory syndrome coronavirus fusion inhibitor. Nat Commun 5:3067. https://doi.org/10.1038/ncomms4067

Mackay IM, Arden KE (2015) Middle East respiratory syndrome: an emerging coronavirus infection tracked by the crowd. Virus Res 202:60-88. https://doi.org/10.1016/j.virusres.2015.01.021

Mateus J, Grifoni A, Tarke A et al (2020) Selective and cross-reactive SARS-CoV-2 T cell epitopes in unexposed humans. Science 370:89-94. https://doi.org/10.1126/science.abd3871

Mou H, Raj VS, van Kuppeveld FJM et al (2013) The receptor binding domain of the new Middle East respiratory syndrome coronavirus maps to a 231-residue region in the spike protein that efficiently elicits neutralizing antibodies. J Virol 87:9379-9383. https://doi.org/10.1128/JVI.01277-13

Ng KW, Faulkner N, Cornish GH et al (2020) (2020) Pre-existing and de novo humoral immunity to SARS-CoV-2 in humans. BioRxiv 05(14):095414. https://doi.org/10.1101/2020.05.14.095414

Rabets A, Bila G, Grytsko R et al (2020) (2020) Development of antibodies to pan-coronavirus spike peptides in convalescent COVID-19 patients. MedRxiv 08(20):20178566. https://doi. org/10.1101/2020.08.20.20178566

Walls AC, Tortorici MA, Snijder J et al (2017) Tectonic conformational changes of a coronavirus spike glycoprotein promote membrane fusion. Proc Natl Acad Sci USA 114:11157-11162. https://doi.org/10.1073/pnas.1708727114

Wang N, Shi X, Jiang L et al (2013) Structure of MERS-CoV spike receptor-binding domain complexed with human receptor DPP4. Cell Res 23:986-993. https://doi.org/10.1038/cr.2013.92

Wrapp D, Wang N, Corbet KS et al (2020) Cryo-EM structure of the 2019-nCoV spike in the prefusion conformation. Science 367:1260-1263. https://doi.org/10.1126/science.abb2507

Xia S, Liu Q, Wang Q et al (2014) Middle East respiratory syndrome coronavirus (MERS-CoV) entry inhibitors targeting 
spike protein. Virus Res 194:200-210. https://doi.org/10.1016/j. virusres.2014.10.007

Xia S, Yan L, Xu W et al (2019) A pan-coronavirus fusion inhibitor targeting the HR1 domain of human coronavirus spike. Sci Adv 5:eaav4580. https://doi.org/10.1126/sciadv.aav4580

Xia S, Liu M, Wang C et al (2020) Inhibition of SARS-CoV-2 (previously 2019-nCoV) infection by a highly potent pan-coronavirus fusion inhibitor targeting its spike protein that harbors a high capacity to mediate membrane fusion. Cell Res 30:343-355. https ://doi.org/10.1038/s41422-020-0305-x

Yaqinuddin A (2020) Cross-immunity between respiratory coronaviruses may limit COVID-19 fatalities. Med Hypotheses 144:110049. https://doi.org/10.1016/j.mehy.2020.110049
Yuan Y, Cao D, Zhang Y et al (2017) Cryo-EM structures of MERS$\mathrm{CoV}$ and SARS-CoV spike glycoproteins reveal the dynamic receptor binding domains. Nat Commun 8:15092. https://doi. org/10.1038/ncomms 15092

Publisher's Note Springer Nature remains neutral with regard to jurisdictional claims in published maps and institutional affiliations. 